\title{
Estimation of influenza and severe acute respiratory illness incidence (burden) in three provinces of the Islamic Republic of Iran, 2012 and 2013
}

M. Gouya ${ }^{1}$, F. Rezaei ${ }^{1}$, A. Haghdoost ${ }^{2}$, M. Nabavi ${ }^{1}$, K. SeifFarahi ${ }^{3}$, E. Mostafavi ${ }^{4}$, T. Mokhtari Azad ${ }^{5}$, H. Akbari $^{1}$, M. Soroush ${ }^{6}$, H. Riazi ${ }^{7}$, E. Bitaraf ${ }^{7}$, M. N. Dadras ${ }^{7}$, H. Barati ${ }^{8}$, H. Shakoori ${ }^{8}$, J. Bathaei ${ }^{9}$, M. Rezvani ${ }^{10}$ and P. Hemmati $^{7}$

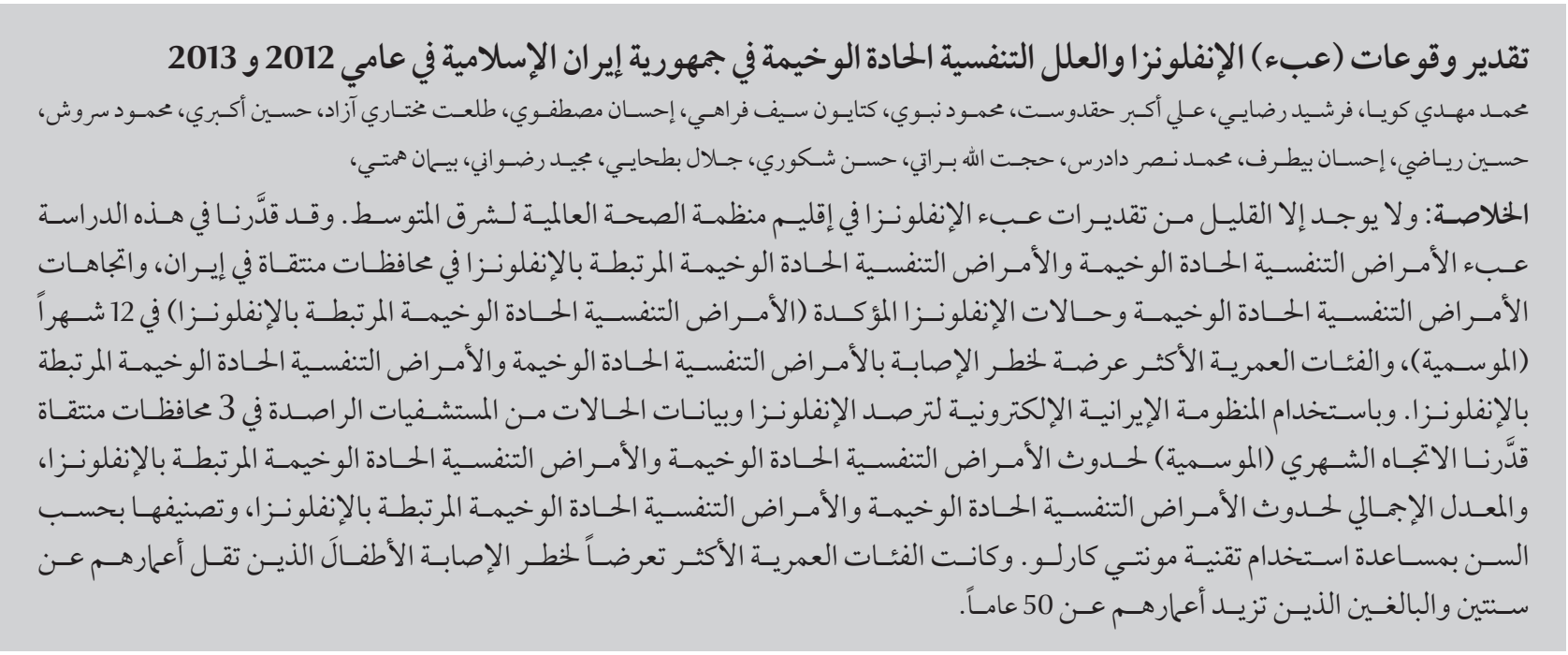

ABSTRACT There are few estimates of influenza burden in the WHO Region for the Eastern Mediterranean. In this study we estimated the burden of severe acute respiratory infection (SARI) and influenza-associated SARI (F-SARI) in selected provinces of Islamic Republic of Iran, the trends of SARI and confirmed cases of influenza (F-SARI) over 12 months (seasonality), and the age groups most at risk. Using the electronic Iranian influenza surveillance system and data of cases in sentinel hospitals of 3 selected provinces, we estimated the monthly trend (seasonality) of incidence for SARI and F-SARI, overall incidence of SARI and F-SARI and their disaggregation by age with the aid using the Monte Carlo technique. The age groups most at-risk were children aged under 2 years and adults older than 50 years.

Estimation de l'incidence (charge) de la grippe et de l'infection respiratoire aiguë sévère en République islamique d'Iran pour 2012 et 2013

RÉSUMÉ Peu d'estimations sont disponibles sur la charge de la grippe dans la Région OMS de la Méditerranée orientale. Dans la présente étude, nous avons estimé la charge de morbidité due aux infections respiratoires aiguës sévères (IRAS) et aux IRAS associés à la grippe dans certaines provinces de la République islamique d'Iran, ainsi que les tendances des IRAS et des cas de grippe confirmés sur 12 mois (saisonnalité), et les groupes d'âge les plus exposés. En utilisant le système électronique iranien de surveillance de la grippe et les données relatives aux cas des hôpitaux sentinelles de trois provinces sélectionnées, nous avons estimé la tendance mensuelle (saisonnalité) de l'incidence des IRAS et des IRAS dus à la grippe, I'incidence globale des IRAS et des IRAS dues à la grippe et leur ventilation par âge grâce à la méthode de Monte Carlo. Les groupes d'âge les plus exposés au risque étaient les enfants de moins de 2 ans et les adultes de plus de 50 ans.

${ }^{7}$ Iranian Center for Communicable Disease Control. ${ }^{2}$ Kerman University of Medical Sciences and Health Services, Kerman, Islamic Republic of Iran. ${ }^{3}$ Iran University of Medical Sciences and Health Services, Tehran, Islamic Republic of Iran. ${ }^{4}$ Epidemiology Department, Pasteur Institute of Iran, Tehran, Islamic Republic of Iran. ${ }^{5}$ National Influenza Center, Tehran University of Medical Sciences and Health Services, Tehran, Islamic Republic of Iran. ${ }^{6}$ Department of Surveillance. ${ }^{7}$ IT Center, Ministry of Health and Medical Education, Tehran, Islamic Republic of Iran. ${ }^{8}$ Department of Disease Control and Prevention, Deputy for Public Health Affairs, Karaj University of Medical Sciences and Health Services, Islamic Republic of Iran. ${ }^{9}$ Department of Disease Control and Prevention, Deputy for Public Health Affairs, Hamedan University of Medical Sciences and Health Services, Islamic Republic of Iran. ${ }^{10}$ Department of Disease Control and Prevention, Deputy for Public Health Affairs, Zanjan University of Medical Sciences and Health Services, Islamic Republic of Iran. (Correspondence to P. Hemmati: hemmatipayman@yahoo.com).

Received: 28/04/2016; accepted: 08/08/2016 


\section{Introduction}

Worldwide, acute lower respiratory infection is the second commonest cause of morbidity and the third commonest cause of mortality in all age groups (1). A significant proportion of the global burden of acute lower respiratory infection, especially in children and the elderly, is attributable to influenza and respiratory syncytial virus (2).

In 2003, it was estimated that in the United States of America annual influenza epidemics resulted in an average of 610660 life-years lost, 3.1 million hospitalized days, 31.4 million outpatient visits, over 334000 hospitalizations and approximately 41000 deaths (3). Direct medical costs averaged US\$ 10.4 billion and lost earnings due to illness and loss of life was estimated at US $\$ 16.3$ billion annually. The total annual economic burden of influenza was estimated to be about US\$ 87.1 billion.

Current global estimates indicate that seasonal influenza affects $5-10 \%$ of the world's population annually, resulting in 250000 to 500000 fatal cases $(4,5)$. Nair et al. estimated that influenza was the second most commonly identified pathogen worldwide in children with acute lower respiratory infection $(5,6)$.

However, in many countries such estimates are rare, especially in the World Health Organization (WHO) Region for the Eastern Mediterranean. The influenza $\mathrm{A}(\mathrm{H} 1 \mathrm{~N} 1)$ pdm09 pandemic highlighted the necessity for reliable estimates of disease burden for severe acute respiratory illness (SARI) and influenza-associated SARI (F-SARI) in all countries and regions of the world. Many countries have established sentinel sites for influenza epidemiological surveillance. The data captured from sentinel sites have been used by WHO Member States to estimate disease burden at the national level and also to compare with other countries.
The fact that many emerging and re-emerging diseases classified under the International Health Regulations (IHR 2005) (7) are of an acute respiratory nature, e.g. severe acute respiratory syndrome (SARS), Middle East respiratory syndrome corona virus (MERS$\mathrm{CoV}$ ), and novel influenza pathogens such as $\mathrm{H} 5 \mathrm{~N} 1$ and $\mathrm{H} 7 \mathrm{~N} 7$, is a strong reminder of the necessity of strengthening surveillance systems for acute respiratory infections and influenza in all WHO Member States, which in turn will enable countries to carry out moreaccurate estimations of the SARI and F-SARI burden.

Having access to a fully electronic influenza surveillance system and to coordinated electronic data for SARI and F-SARI countrywide, the Iranian Center for Communicable Disease Control recognized the necessity of performing in-depth analyses of pooled data from the Iranian Influenza Surveillance System (IISS) in selected provinces in 2012 and 2013. Prior to this, to our knowledge, no proper study on SARI and information on the burden of influenza had been carried out in the Islamic Republic of Iran.

The objectives of our study were to:

- estimate the burden of SARI and FSARI in selected provinces of Iran;

- review the trends for SARI and confirmed cases of F-SARI over 12 months (seasonality);

- determine the age groups most at risk of SARI and F-SARI.

- The study had the following advantages:

- providing comparable assessment of SARI and the proportion attributable to F-SARI, information which will assist in informed decision-making and planning for augmentation of vaccine manufacturing or supply capacity and proper estimation of required quantities of antivirals for reducing influenza-related morbidity and mortality;
- assisting stakeholders, e.g. donor nongovernmental organizations, United Nations agencies, WHO and countries, in prioritizing public health research investments and interventions when allocating scarce resources;

- guiding the health system and other sectors in needs assessment and better planning for services before and during outbreaks/epidemics of acute respiratory infections;

- providing baseline incidence information for comparison of influenza epidemics in different years, which can help identify emerging events such as influenza pandemics or acute respiratory infections with novel agents.

\section{Methods}

\section{Setting}

The Islamic Republic of Iran is the second largest country in the Middle East and, with 78.4 million inhabitants, it is the world's 17 th most populous nation.

The influenza surveillance system was developed in 2004. The first version was paper-based. Due to the shortcomings of this, the Iranian Center for Communicable Disease Control upgraded the system into a fully electronic countrywide version, the IISS, in 2009. This has three integrated components:

- influenza-like illness (ILI) sentinelbased surveillance as an early warning component;

- case-based (patient-centred) surveillance, which captures epidemiologic and clinical information of sampled cases in hospitals, in selected ambulatory health centres (sentinel sites) and in closed communities in the case of an outbreak of acute respiratory illness;

- laboratory-based surveillance, which collects laboratory results of sampled cases.

The IISS has been added as part of the electronic health record system 
of the Ministry of Health and Medical Education. Advantages resulting from IISS include: a unique database and coordinated countrywide surveillance system for influenza; monitoring trends and responses to influenza outbreaks at district, provincial and national levels; creating automated analysis reports to support public health authorities and assist timely evidence-based decisions; enabling higher levels of the health system to supervise and evaluate the performance of lower levels; and providing feedback to lower levels. The SARI surveillance identifies cases which have been admitted to hospital for medical care due to respiratory symptoms (fever, cough and severe symptoms which require hospitalization e.g. dyspnoea). In accordance with Iranian Center for Communicable Disease Control policy, it was planned that all SARI cases in hospital be sampled, but for a variety of reasons some cases may not have been sampled. To calculate the sampling fraction, we used IISS data to count the numbers of SARI cases in the sentinel hospitals of selected provinces. In the meantime, we asked the targeted provinces to report the number of pneumonia cases recorded by the admission office of these hospitals. By comparing these two values, we were able to calculate the proportion of SARI cases from whom clinical specimens were collected for laboratory confirmation of influenza, i.e. the sampling fraction, which was later used to estimate the true number of F-SARI cases.

In this study we used the WHO method and guidelines for estimation of influenza burden (5), which was demonstrated in a WHO workshop held in Egypt in December 2012. We set our key sources of data in SARI sentinel hospitals (not a national model). For the first step, the hospital with the greatest number of admitted SARI cases is selected as the sentinel hospital of a target district, then the sentinel hospital and other hospitals in the same district are listed in a worksheet. After that the number of cases of SARI admitted to the sentinel hospital and other hospitals are entered into the worksheet and disaggregated by age. The proportion of SARI cases admitted to the sentinel hospital compared with the total number of SARI cases in all district hospitals is calculated over at least 1 year (12 months). Using this information, as well as the mid-year population for each age group in the district, the catchment area of the sentinel hospital was estimated for each age group. Finally, using the number of SARI and F-SARI cases in the sentinel hospital (the numerator) for each month and the catchment area population (2012) as denominator, the monthly and annual incidences of SARI and F-SARI were estimated.

\section{Patient data collection}

The Iranian Center for Communicable Disease Control selected the threeprovinces with the greatest number of SARI and F-SARI cases registered in IISS, Western Azerbaijan, Alborz, and Hamedan. The hospital in each province with the greatest numbers of SARI cases was selected as the SARI sentinel site, and used for the estimation of incidence of influenza and SARI according to the WHO methodology. We sent the translated version of the $\mathrm{WHO}$ manual for influenza burden estimation to all provinces. The selected provinces were asked to collect the necessary data for influenza burden estimation using worksheets No. 5 through 7 (5). Worksheet number 5 is a tool for estimating the proportion of pneumonia cases (from the catchment area) accessing a SARI sentinel hospital for treatment. Using this tool, we first entered the total number of SARI cases during the previous 12 months in all hospitals of the catchment area, including the sentinel hospital. Then we computed the proportion of SARI cases (accessing sentinel sites for treatment). This helped in estimating the proportion of SARI cases in different age groups in the catchment area who went to the sentinel hospital for consultation.

The selected provinces, Western Azerbaijan, Alborz and Hamedan, are all located in north-western and western mountainous parts of the country, with mainly cold weather during at least 7-8 months of the year All three provinces have comparable altitude and weather conditions (Table 1), both of which affect influenza virus activity and seasonality. According to the WHO manual, the data collected were of appropriate quality (influenza case finding was expected to be around $10-20 \%$ of SARI cases during the cold seasons) $(5,8-10)$ and the definitions of ILI and SARI cases had been followed.

Another factor which made these three provinces more appropriate compared with other provinces was the

\begin{tabular}{lccccc}
\hline Table 1 Altitude, minimum and maximum temperature of selected cities in the three targeted provinces \\
City/province & $\begin{array}{c}\text { Altitude } \\
(\mathbf{m})^{\mathbf{a}}\end{array}$ & $\begin{array}{c}\text { Minimum } \\
\text { average annual } \\
\text { temperature }\left({ }^{\circ} \mathbf{C}\right)\end{array}$ & $\begin{array}{c}\text { Maximum } \\
\text { average annual } \\
\text { temperature }\left({ }^{\circ} \mathbf{C}\right)\end{array}$ & $\begin{array}{c}\text { No. of frosty } \\
\text { days during the year }\end{array}$ & $\begin{array}{c}\text { Population } \\
\text { according to } \\
2011 \text { census }^{\mathrm{b}}\end{array}$ \\
Karaj city/Alborz & 1300 & 1.2 & 26.0 & 50 & 2412513 \\
Hamedan/Hamedan & 1741 & 3.3 & 19.1 & 111 & 651821 \\
Mahabad/Western Azerbaijan & 1320 & 4.2 & 19.3 & 99 & 215489 \\
\hline
\end{tabular}

a Source: https://en.wikipedia.org/wiki/Karaj; https://en.wikipedia.org/wiki/Hamadan; https://en.wikipedia.org/wiki/Urmia.

${ }^{b}$ Source: Statistical Centre of Iran (http://www.sci.org.ir/SitePages/report_90/ostani/ostani_population_report_final_permision.aspx). 
number of SARI cases recorded in IISS being equal to or lower than the number reported by the sentinel hospitals. After conducting a training workshop and subsequently providing sufficient practice and feedback, the Center for Communicable Disease Control asked the three selected provinces to carefully review their own data and again fill out worksheets 5, 6 and 7 from the WHO manual (5). The worksheets helped the selected provinces to gather the following data:

- total number of cases of pneumonia (SARI) during 12 months managed in the sentinel hospital and other hospitals in the selected district of the province, distributed by age;

- population of the selected district using data from the population census for different age groups in order to calculate the catchment area population of the sentinel hospital;

- total number of F-SARI cases (positive for all types and subtypes of human influenza viruses) in the sentinel hospital distributed over 12 months;

- number of sampled SARI cases from the sentinel hospitals (recorded on the electronic platform, i.e. IISS);

- total number of SARI cases (from all sentinel hospital wards which admit SARI cases), including cases not tested for influenza or testing negative for influenza based on laboratory results (data were acquired from sentinel hospitals not from IISS in order to consider and calculate correction index for cases not sampled and entered into IISS);

- correction index: calculated using SARI cases captured by IISS divided by SARI cases reported by sentinel hospital; this index was calculated for each age group then applied to correct the number of F-SARI cases (this correction could, to some extent, compensate for underestimation of influenza cases by influenza surveillance).
Considering the geographical proximity and seasonal similarities of the three target provinces, it was possible to pool the data. This is useful as it improves the reliability of burden estimates.

\section{Ethics}

The study procedures were reviewed by relevant ethical committees in the three selected provinces. Considering the anonymity of cases and the use of aggregated data, no ethical problems were observed while carrying out this study.

\section{Specimens and testing of patients}

Pharyngeal swabs were taken from suspected cases of SARI at the hospitals, and the specimens were placed in viral transport medium (UTM transport medium, Copan Diagnostics) and shipped to influenza reference laboratories in Tabriz, Tehran and Kermanshah (storage temperature $4-8{ }^{\circ} \mathrm{C}$ ). The specimens were tested using polymerase chain reaction to detect influenza viruses $\mathrm{A} / \mathrm{H} 1 \mathrm{~N} 1, \mathrm{~A} / \mathrm{H} 3 \mathrm{~N} 2$ and $\mathrm{B}$.

\section{Data analysis}

The SARI and F-SARI data for the threetargeted provinces were pooled and the correction indices were calculated for each age subgroup. The pooled data were entered into the Iranian Center for Communicable Disease Control software for estimation of SARI and influenza burdens (cumulative incidence).

Using the number of SARI and FSARI cases in the three sentinel hospitals (numerator) over 12 months and the mid-year catchment area populations for the same year (2013) as the denominator, the monthly and annual incidence of both SARI and F-SARI were estimated.

First a point estimate was calculated for cumulative incidence of SARI and F-SARI for each age group and for seasonality (cumulative incidence for each month of the year), then, using the
Monte Carlo technique and simulating 1000 scenarios, the $95 \%$ confidence interval (CI) for incidence of SARI and F-SARI was calculated for the different age groups.

One of prerequisites for the application of Monte Carlo modelling is defining a minimum and maximum for the case finding sensitivity rate. Sensitivity rate is defined as the proportion of SARI patients who reside in the catchment area of the sentinel hospital and come to the same sentinel hospital for admission. Minimum and maximum figures for sensitivity were estimated by influenza surveillance officers in the 3 provinces. The mean of the estimated sensitivity rates for the 3 provinces was used for pooled data. The average minimum and maximum sensitivity rates for F-SARI in 3 provinces were 0.5 and 0.7 , while for SARI cases they were 0.6 and 0.95 , respectively.

In this study, we considered the start of the influenza season as the beginning of the 12-month study, so the last 2 months of 2012 and the first 10 months of 2013 were considered.

\section{Results}

Over the period November 2012 October 2013, 8068 SARI cases were registered by the IISS countrywide (3939 males and 4129 females), and 1022 were recorded from the sentinel hospitals of the 3 provinces covered in this study; thus, the proportion of cases from the 3 sentinel sites was $12.7 \%$. The distribution of cases recorded in IISS for each district and of those reported by sentinel hospitals are reflected in Table 2.

Among the F-SARI cases, 68\% were A/H1Nlp, 28\% were influenza type $\mathrm{B}$ and $4 \%$ were $\mathrm{A} / \mathrm{H} 3 \mathrm{~N} 2$. There was a seasonal pattern for F-SARI, with several peaks in the cold season (Figure 1). The seasonal peaks for SARI cases occurred around May-June. The age groups most at-risk were children under 


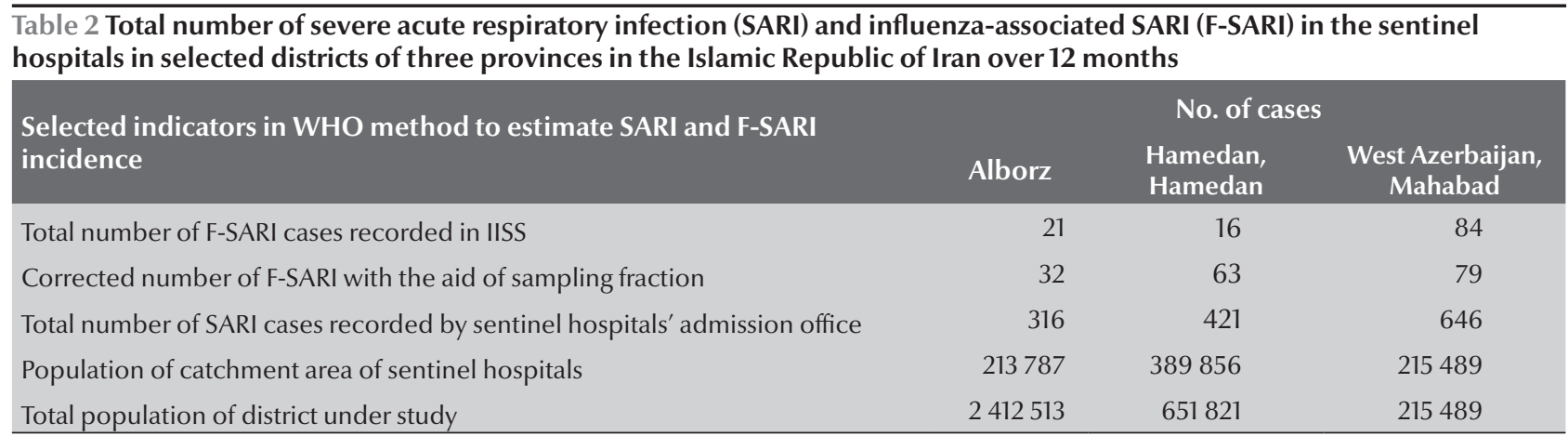

IISS = Iranian Influenza Surveillance System.

2 years $(75.2 \%)$ and adults older than 50 years $(20.36 \%)$ (Figure 2 ).

The mean annual incidence for all age groups for F-SARI and SARI in Alborz province was 15.0 and 147.8 per 100000 population respectively; in Hamedan province the values were 9.3 and 62.7, and in West Azerbaijan 36.7 and 299.8 per 100000 population respectively.

Using the Monte Carlo simulation, the incidence of SARI and F-SARI for all age groups in Alborz province was estimated at 194.3 (95\% CI: $138.4-$ 262.9,) and 25.6 (95\% CI: 14.9-38.1); in Hamedan province it was 82.3 (95\% CI: $60.2-111.0)$ and 15.9 (95\% CI:
8.6-23.8), while in West Azerbaijan province it was 393.9 (95\% CI: $282.7-$ 526.5) and 61.3 (95\% CI: 35.8-92.6) per 100000 population respectively. Using pooled data from the three provinces, the overall incidence for SARI and F-SARI for all age groups was calculated at 187.6 (95\% CI: 137.1-247.8) and 29.0 (95\% CI: 16.8-43.8) per 100000 population respectively.

\section{Discussion}

The trend for the incidence of SARI follows a sinusoidal pattern in cold provinces of the Islamic Republic of Iran,

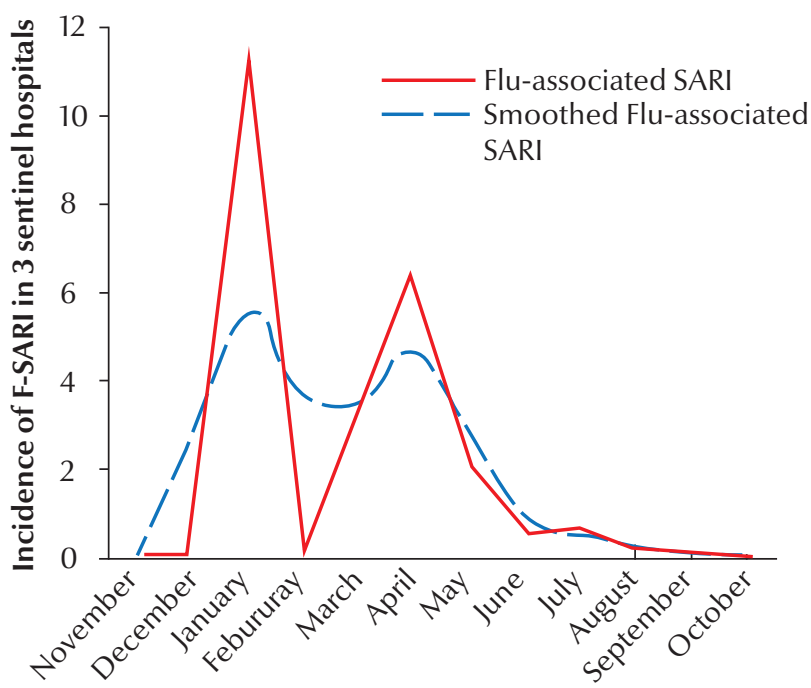

Figure 1 Seasonality of influenza-associated SARI (F-SARI) (cases per 100000 population), November 2012-October 2013 (Source: pooled data from 3 provinces in the Islamic Republic of Iran)

with a downward trend and a minimum during the warm months, June-September. Then it takes an upward trend during the cold season, October-May. For F-SARI there are two or three peaks in cold provinces. The first peak, the main wave, occurs around December to January, while the second occurs around March. Multiple waves in each influenza season have been reported by other researchers $(11,12)$. There is a difference between F-SARI and SARI: between June and late October influenza virus activity approaches zero, while SARI has a minimum level of occurrence, even during the warm season. The downward trend in the activity of F-SARI observed during this period of the year along with the concurrent increased activity of SARI suggests the possible activity of another respiratory pathogen (other than influenza) which merits investigation in the future studies. The differences between SARI and FSARI incidences in the three provinces may be attributable to different levels of immunity among these populations or different levels of interventions such as following respiratory protocols or vaccination policies for at-risk groups. Based on our experience, the performance of public health systems in the three districts is very different, which implicitly indicates the effectiveness of public health measures in modifying the impact of seasonal influenza. However, testing these hypotheses would require further study and was beyond the objectives of the current study. 


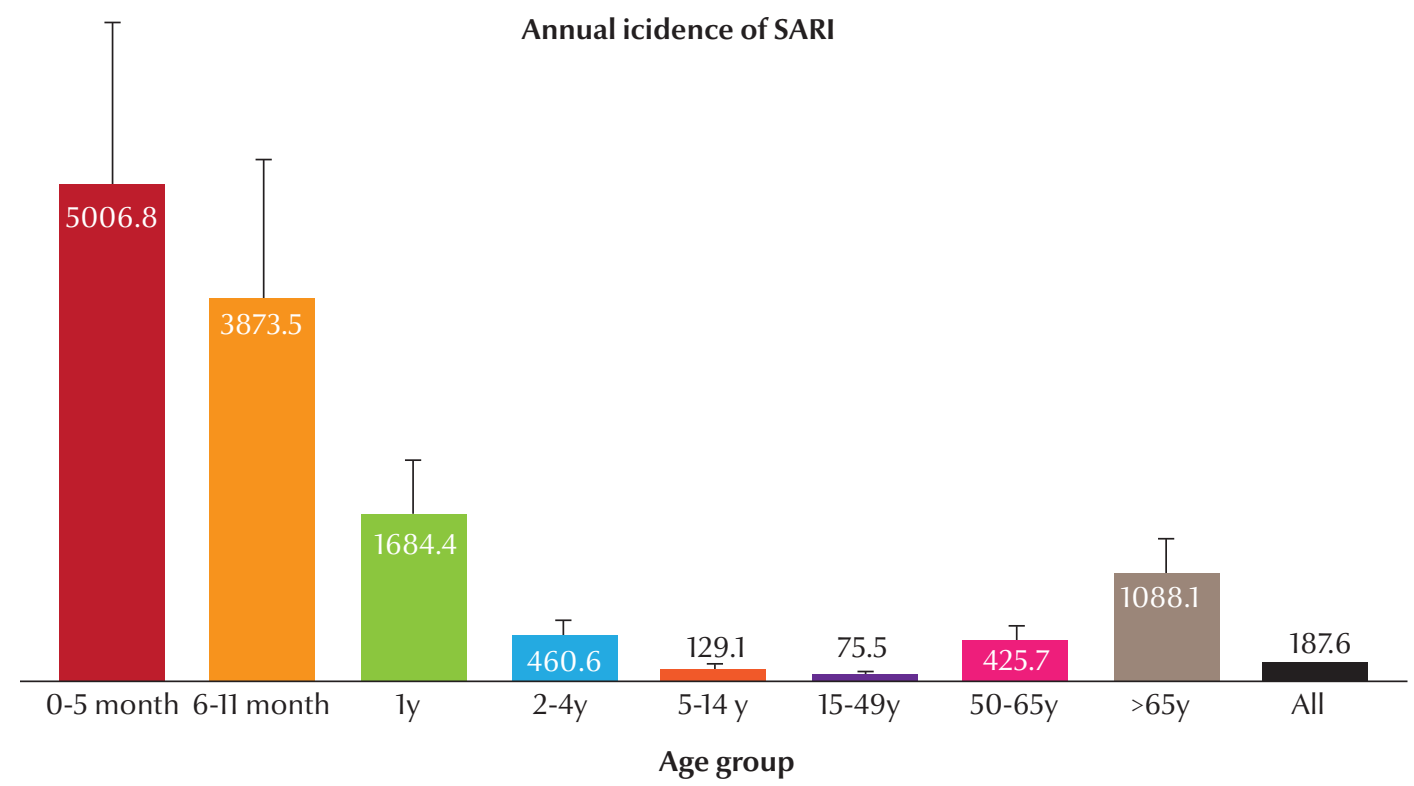

Figure 2 Estimated incidence of severe acute respiratory infection (SARI) (cases per 100000 population) distributed by age group, November 2012-October 2013 (Source: pooled data from sentinel sites in 3 provinces in the Islamic Republic of Iran using Monte Carlo simulation)

Those most at risk of SARI and FSARI, were children under 2 years of age and those over 50 years. Similar findings have been reported in studies by Le et al. (13), Fuller et al. (14), Burmaa et al. (15) and Seo et al. (16). In two American studies, the age group most at risk for fatality was reported as those older than 65 years $(17,18)$. The overall incidence of SARI and F-SARI in our study was estimated at 187.6 and 29.0 per 100000 population, respectively. In a Peruvian study by Tinocco et al. (19) the annual incidence of influenza per 100000 population in different age groups was calculated as: $<5$ years, 277; 5-17 years, 225 ; $18-49$ years, 74 ; 50-64 years, 65; and > 65 years, 46 . We estimated the annual incidences for different age groups as: $<5$ years, 492 ; $5-14$ years, $1 ; 15-49$ years, 23 ; 50-64 years, 86 ; and $>65$ years, 47 . From these estimates, it seems that there is a higher incidence in the Iranian population younger than 5 years compared with the Peruvian population, a lower incidence among adolescents and adults and comparable incidence among people older than 50 years.

\section{Limitations}

There were some limitations in this study. The trend for SARI and F-SARI was only studied in three mountainous provinces. When quality data are available in other provinces with different weather conditions, a more comprehensive study should be performed. As the electronic countrywide platform for influenza surveillance (IISS) is new in this country, we expect to improve its quality over a broader geographical area, then similar studies can be conducted to estimate SARI and F-SARI incidence in provinces with different weather and geographical situations.

The data in this study were limited to a 12 -month period. Through monitoring data over several consecutive years, a better epidemiological picture of SARI and seasonal influenza could be described, which would be more helpful in detecting unusual patterns of activity in the case of emergent novel respiratory pathogens such as those responsible for pandemic influenza. We were unable to estimate SARI and influenza burden disaggregated by sex due to limited data in each subgroup. In a few cases in the target provinces the sentinel hospitals reported fewer cases than those recorded in IISS. This could be due to misunderstandings regarding the definition of SARI and its difference from the definition of pneumonia by hospital staff while there was good understanding of the definition of SARI among public health staff who captured and recorded SARI cases in IISS.

Although we applied the SARI definition to detect suspected cases of influenza, which is more sensitive than the routine pneumonia definition, we may still have underestimated the actual burden, since not all influenza cases are recorded as SARI in hospital records but sometimes recorded as myocardial infarction or exacerbation of comorbidities (9).

In many studies, the F-SARI incidence was only reported in children aged under 5 years, while in our study we estimated the incidence in all age groups $(15,16)$.

\section{Conclusion and recommendations}

The tool developed by Iranian Center for Communicable Disease Control 
enabled the rapid estimation of the burden of SARI and F-SARI in every district or province for which data were available. In some provinces where there was a discrepancy between SARI cases recorded by hospitals and cases registered in the public health sector in IISS, it is possible to provide feedback and improve the performance of hospital surveillance in the future.

According to a number of studies, between $6.6 \%(20)$ and $30.0 \%(21-23)$ of SARI cases would progress into very severe forms that need care in an intensive care unit. These estimates could be informative for decision-makers at provincial and national levels in assessing the requirements for intensive care unit equipment. Another potential application could be estimation of the number of vaccines required to prevent epidemics of seasonal influenza, especially in high-risk groups, as well as estimation of the economic burden of influenza and the effectiveness of measures such as public information/communication.
The IISS initiative employs and developing technology in surveillance systems. It has been providing feedback to provinces and helping identify issues in the influenza programme. It has also helped the Iranian Center for Communicable Disease Control and the provinces in continuously strengthening their influenza surveillance. Thus, it is expected that over the next few years more provinces will improve the quality of their data, which will be valuable in improving the estimation of influenza and SARI burdens.

The results of this study are mainly applicable for SARI and influenza burden estimations. However, there are other observations and findings pointing to potential associations between the ILI, SARI and F-SARI syndrome. The exploration of such associations could be useful as an early warning signal before or in the very early stages of the emergence of an epidemic of influenza or other respiratory agents. It is recommended to test this hypothesis in further studies.

\section{Acknowledgements}

We would like to thank Dr Magsoud Ebrahimnezhad, Urmia University of Medical Sciences and Health Services, for his constructive feedback to improve the performance of IISS. We would also like to thank Fatemeh Abedi Astaneh, Senior Officer, Disease Control and Prevention, Deputy for Public Health Affairs, Qom University of Medical Sciences and Razieh Khajehkazemi, PhD student at Kerman University of Medical Sciences, Islamic Republic of Iran..

Funding: This research has been supported by the Iranian Center for Communicable Disease Control, Undersecretary for Public Health, Ministry of Health and Medical Education, Tehran, Islamic Republic of Iran.

Competing interests: None declared.

\section{References}

1. Mathers C, Fat DM, Boerma JT. The global burden of disease: 2004 update. Geneva, Switzerland: World Health Organization; 2008.

2. Graham NMH. The epidemiology of acute respiratory infections. In: Nelson KE, Williams CM, Graham NMH, editors. Infectious disease epidemiology: theory and practice. Gaithersburg (Maryland): Aspen Publishers; 2001. pp. 439-76.

3. Molinari NM, Ortega-Sanchez IR, Messonnier ML, Thompson WW, Wortley PM, Weintraub E, et al. The annual impact of seasonal influenza in the US: measuring disease burden and costs. Vaccine. 2007;25(27):5086-96.

4. Influenza (Seasonal). Geneva: World Health Organization; 2014 (Fact sheet No. 211) (http://www.who.int/mediacentre/ factsheets/fs211/en, accessed 16 March 2016)

5. Nair H, Campbell H, Mounts A. A manual for estimating disease burden associated with seasonal influenza in a population. Geneva: World Health Organization; 2012.

6. Nair H, Brooks WA, Katz M, Roca A, Berkley JA, Campbell H, et al. Global burden of respiratory infections due to seasonal influenza in young children: a systematic review and metaanalysis. Lancet. 2011;378(9807):1917-30.

7. World Health Organization. International Health Regulations (2005). 2nd ed. Geneva: World Health Organization; 2008.

8. Savy V, Ciapponi A, Bardach A, Glujovsky D, Aruj P, Mazzoni A, et al. Burden of influenza in Latin America and the Caribbean: A systematic review and meta-analysis. Influenza Other Respi Viruses. 2012;7(6):1017-32.
9. Yu H, Huang J, Huai $Y$, Guan $X$, Klena J, Liu S, et al. The substantial hospitalization burden of influenza in central China: Surveillance for severe, acute respiratory infection, and influenza viruses, 2010-2012. Influenza Other Respi Viruses. 2013;8(1):53-65.

10. <eref $>10$. California influenza and other respiratory disease surveillance for week 11 (March 9 to March 15, 2014). Sacramento: California Department of Public Health Influenza Surveillance Program; 2014 (http://www.cdph.ca.gov/Healthlnfo/ discond/Documents/Week\%2011\%20-\%20FINAL\%20Report. pdf, accessed 16 March 2016).

11. Ali A, Khowaja AR, Bashir MZ, Aziz F, Mustafa S, Zaidi A. Role of human metapneumovirus, influenza $A$ virus and respiratory syncytial virus in causing WHO-defined severe pneumonia in children in a developing country. PLoS One. 2013;8(9):1-5.

12. He Y, Lin G, Wang Q, Cai X, Zhang Y, Lin C, et al. A 3-year prospective study of the epidemiology of acute respiratory viral infections in hospitalized children in Shenzhen, China. Influenza Other Respi Viruses. 2014;8(4):443-51.

13. Le MN, Yoshida LM, Suzuki M, Nguyen HA, Le HT, Moriuchi $\mathrm{H}$, et al. Impact of 2009 pandemic influenza among Vietnamese children based on a population-based prospective surveillance from 2007 to 2011. Influenza Other Respi Viruses. 2014;8(4):389-96.

14. Fuller JA, Summers A, Katz MA, Lindblade KA, Njuguna $H$, Arvelo W, et al. Estimation of the national disease burden of influenza-associated severe acute respiratory illness in Kenya and Guatemala: a novel methodology. PLoS One. 2013;8(2): $1-11$ 
15. Burmaa A, Kamigaki T, Darmaa B, Nymadawa P, Oshitani H. Epidemiology and impact of influenza in Mongolia, 2007-2012. Influenza Other Respi Viruses. 2014;8(5):530-7.

16. Seo YB, Song JY, Cheong HJ, Cho YD, Wie S, Jeong HW, et al Hospital-based influenza morbidity and mortality surveillance system for influenza-like illnesses: a comparison with national influenza surveillance systems. Influenza Other Respi Viruses. 2013;8(1):17-20.

17. Thompson MG, Shay DK, Zhou H, Bridges CB, Cheng PY, Burns $M A$, et al. Estimates of deaths associated with seasonal influenza - United States, 1976-2007. MMWR Morb Mortal Wkly Rep. 2010 Aug;59(33):1057-62. [cited 2016 March 16] (http:// www.cdc.gov/mmwr/PDF/wk/mm5933.pdf).

18. Cheng P, Palekar R, Azziz-Baumgartner E, Iuliano D, Alencar AP, Bresee J, et al. Burden of influenza-associated deaths in the Americas, 2002-2008. Influenza Other Respi Viruses. 2015 Aug;9 Suppl. 1:13-21.

19. Tinoco YO, Azziz-Baumgartner E, Razuri H, Kasper MR, Romero $\mathrm{C}$, Ortiz E, et al. A population-based estimate of the economic burden of influenza in Peru, 2009-2010. Influenza Other Respi Viruses. 2016; 10(4):301-9.
20. Tramuto F, Maida CM, Bonura F, Perna AM, Puzelli S, De Marco MA, et al. Surveillance of hospitalized patients with influenzalike illness during pandemic influenza A(H1N1) season in Sicily, April 2009-December 2010. Euro Surveill. 2011 Sep;16(35):1-8 [cited 2016 March 16] (http://www.eurosurveillance.org/ ViewArticle.aspx?Articleld=19957).

21. Santa-Olalla Peralta P, Cortes-García M, Vicente-Herrero M, Castrillo-Villamandos C, Arias-Bohigas P, Pachon-del Amo I, et al. Risk factors for disease severity among hospitalized patients with 2009 pandemic influeza A (H1N1) in Spain, April - December 2009. Euro Surveill. 2010 Sep;15(38):1-9 [cited 2016 March 16] (http://www.eurosurveillance.org/ViewArticle. aspx?Articleld=19667)

22. Kelly PM, Kotsimbos T, Reynolds A, Wood-Baker R, Hancox B, Brown SG, et al. FluCAN 2009: initial results from sentinel surveillance for adult influenza and pneumonia in eight Australian. Med J Aust. 2011 Feb;194(4):169-74.

23. Mytton OT, Rutter PD, Donaldson LJ, Influenza A. H1N1)pdm09 in England, 2009 to 2011: a greater burden of severe illness in the year after the pandemic than in the pandemic year. Euro Surveill. 2012 Apr;17(14):1-9 [cited 2016 March 16] (http://www. eurosurveillance.org/ViewArticle.aspx?Articleld=20139). 